\title{
Social and Distributional Effects of Public Transport Fares and Subsidy Policies Case of Madrid, Spain
}

\author{
Paola Carolina Bueno Cadena, Jose Manuel Vassallo, Israel Herraiz, \\ and Manuel Loro
}

\begin{abstract}
Despite the widespread implementation of urban transport subsidies in many cities, there are still only a limited evaluation of the equity of these policies and scarce quantitative assessment of their distributional incidence. This research contributes to filling this gap by developing a practical approach to evaluate the impact of fare subsidization on vertical equity. This paper implements a two-step methodology. First, two main indicators were developed to measure the social impact of the travel pass, a highly subsidized fare, to determine the effectiveness of the policy in reaching lower-income citizens. Second, by using the latest disaggregated data from a transportation survey, in Madrid, Spain, a multiple regression model revealed that travel pass usage (TPU) depended mainly on income level and accessibility to public transport. The results show that the accessibility level has a positive effect on the TPU indicator, whereas income level has a negative influence. Because income level is shown to play the most significant role in influencing public transport use, the subsidy policy associated with the travel pass in the city can be considered progressive, since it effectively targets economically disadvantaged groups. This fact suggests that subsidies for public transport in Madrid tend to favor vertical equity.
\end{abstract}

Despite the increasing interest in equity issues within the concept of sustainability, scholars agree that social impacts and their distributional effects have traditionally received less attention than economic and environmental aspects. One of the drawbacks of including social impacts in the evaluation of public policies is that there is considerable uncertainty as to what a social impact is, how to measure it, and how to evaluate its distributional effects and equity issues (1). Frequently, "transport projects are implemented by using methods such as Cost-Benefit Analysis which makes extensive use of financial costs and benefits without considering social impacts of the project at a disaggregate level" (2). There are today very few standardized methods for evaluating the social and distributional effects of public policies. Knowledge is fragmented across a large number of different disciplines and, consequently, the concept of equity is addressed in the literature from different perspectives.

P. C. Bueno Cadena and J. M. Vassallo, Transport Research Center-TRANSYT, Universidad Politécnica de Madrid, Avenida Profesor Aranguren s/n, Ciudad Universitaria, 28040 Madrid, Spain. I. Herraiz, Department of Applied Mathematics and Computing, and M. Loro, Department of Transport and Territory, Universidad Politécnica de Madrid, Avenida Profesor Aranguren s/n, Ciudad Universitaria, 28040 Madrid, Spain. Corresponding author: P. C. Bueno Cadena, pbueno @caminos.upm.es.

Transportation Research Record: Journal of the Transportation Research Board, No. 2544, Transportation Research Board, Washington, D.C., 2016, pp. 47-54. DOI: 10.3141/2544-06
Much less is known about the consequences of transport policies on equity. The literature on transport policies mainly focuses on economic and environmental rather than social and distributional impacts. As Nuworsoo et al. observed, only a few authors have looked at equity aspects of transit fare policies per se (3). Equity considerations associated with fare policies and the distributional incidence of subsidies have been subjected to very little detailed analysis, as the common perception is that subsidies always have progressive distribution effects (4). Although equity is often the key component in debates on how to allocate public resources, the social and distributional dimensions of public transport subsidies have drawn significantly less attention in the literature (5).

This paper presents a thorough assessment of the social and distributional effects of the public transport fare and subsidy policy in the city of Madrid, Spain. The research is based on a two-step methodology. First, two quantitative indicators formulated from Madrid's Transport Survey are defined and evaluated. Second, this information is used to calibrate a multiple regression model to study the variables explaining the use of the travel pass. By exploring the equity implications of the travel pass approach, the aim is to determine whether this policy provides benefits to the people in most need, namely low-income groups. The results show that the accessibility level has a positive effect on travel pass usage (TPU), whereas income level has a negative influence. Because income level is shown to play the most significant role in influencing public transport use, the subsidy policy associated with the travel pass in the city can be considered progressive, since it effectively targets economically disadvantaged groups. This fact suggests that subsidies for public transport in Madrid tend to favor vertical equity.

This article summarizes current knowledge on the social impacts of transport, especially equity impacts, and presents the characteristics of the city of Madrid and its mobility. The next section outlines the research approach for addressing the concept of social justice in relation to the most highly subsidized fare in Madrid: the travel pass. The results of the evaluation of Madrid's fare policy are then discussed and, finally, conclusions and recommendations for additional research in the field of transport equity are presented.

\section{LITERATURE REVIEW: EQUITY IMPLICATIONS OF TRANSIT FARE POLICIES}

"Equity refers to the distribution of impacts—benefits and costsamong members of the society and whether this distribution is considered appropriate" (6). Equity is closely related to the concept of 
fairness. For a policy to be considered equitable, it should distribute its costs and benefits in a fair way across society (7).

Although the concept of equity is a major premise of transport subsidy policies, the social and distributive dimensions of urban transport subsidies have received scarce attention in the literature. According to Serebrisky et al., despite the many subsidy policies adopted for urban public transport, "there are virtually no quantitative assessments of their distributional incidence, making it impossible to determine if these policies are pro-poor" (5).

Although there are few studies on the distributional incidence of transport subsidy policies, a number of methodological approaches have been developed to analyze the equity effects of urban transport subsidies. Nuworsoo et al. analyzed the five alternative fare proposals for the Alameda-Contra Costa Transit District in California, by paying particular attention to the impact on certain riders (gender, income group, and race) (3). The research acknowledges that certain of the riders' characteristics (such as income, age, and trip purpose) make them more sensitive than others to price changes. The research conducted by Bureau and Glachant, using disaggregated data from the Global Transport Survey, evaluates the distributional effects of alternative urban public transport scenarios in the Paris Region (fare reduction and increase in the speed of public transport) (8). These authors found that the changes were progressive and that low-income populations perceived more benefits from fare reductions than from increases in public transport speed.

There are different opinions and results with regard to the progressiveness of targeting subsidies to the poor. Some researchers have shown that the effect of transit subsidies redistribute income from high-income to low-income classes $(9,10)$, while other authors have concluded that transport subsidies show either a neutral or regressive distributive impact $(5,11)$.

The case of Spain is of particular interest: first, because urban public transport in Spanish cities is highly subsidized (12); second, the public sector is currently facing budget cuts; and third, only a few studies have looked at the equity of transit fare policies. For instance, the redistribution effects of urban transport subsidies at the municipal level throughout the country were analyzed by Asensio et al., who calibrated a model that took into account expenditure on urban public transport (12). From calculations of Gini indexes in different municipalities, these researchers concluded that in general terms urban public transport subsidies in Spain were progressive and had a positive redistributive effect.

In Madrid, there is very little analysis of the distributional incidence of public transport subsidies. There are some studies that examine how users respond to changes in prices and service characteristics in the Madrid metropolitan area (13) and evaluate the impact on revenue with the introduction of the travel pass (14). By estimating demand equations and elasticities and quantifying explanatory variables for the significant rise in public transportation use, it was found "that despite succeeding in reversing the declining patronage trend of public transportation in Madrid, the adoption of these transportation policies tended to require an increasing financial support that was not always available" (14).

A broad analysis of urban public transport policy funding in Madrid looked at the evolution of public transport subsidies between 1995 and 2005 and their relationship with equity aspects (4). On the basis of a simple correlation analysis between the use of the travel pass and income per capita by geographical zone, the authors found that, in the city of Madrid, the lower the income level was, the higher the use of the travel pass was. However, Vassallo et al. pointed out that in the outer zones of the metropolitan area the correlation between the level of income and use of travel passes was almost nonexistent (4).
The contribution of this paper to the literature is that it analyzes whether categorical transport subsidies in the city (preferential fares for groups such as senior citizens and young people) are related to income and provides a methodology to quantitatively evaluate the distributional effect of transit fare subsidy. A further difference with earlier works is that the analysis is not restricted to income and social class at the metropolitan level or the municipal level in the Madrid metropoli$\tan$ area. As far as the authors are aware, there are no previous studies analyzing public transport subsidy policy at a disaggregated level, such as by neighborhoods.

For the purpose of this paper, the term "subsidy" with regard to a monthly pass should not be interpreted as part of programs in which employers provide benefits for employees (e.g., transit pass reimbursements) but as a discount involved in a monthly travel pass in and of itself.

\section{CHARACTERISTICS OF THE CITY OF MADRID AND ITS MOBILITY}

\section{City of Madrid: Overview of Income Distribution}

More than 6 million inhabitants live in the Madrid metropolitan area, which comprises 179 municipalities. About 50\% of the population lives in the city of Madrid, in an area of $607 \mathrm{~km}^{2}$. According to the National Institute of Statistics, the average population density in the city is 5,390 inhabitants per square kilometer. The distribution of residential areas and employment centers gives rise to an urban structure that follows a predominantly monocentric model, with radial trips from satellite settlements to the city center. This structure makes Madrid well suited to public transportation use. Currently, as the 2004 Transport Survey reveals, "there are 6,670,000 motorized trips every working day- $48 \%$ served by public transport and $52 \%$ by car" (Monzón and Guerrero, 15).

The city is split into 21 districts, which are further subdivided into 128 neighborhoods and 2,412 census sections. Unlike the trend in some of the world's cities, the districts and neighborhoods located in the center of Madrid are wealthier than the average for the city. Furthermore, the income distribution within each neighborhood is fairly homogeneous. In other words, low- and high-income neighborhoods do not overlap, and the distribution is not highly skewed. Average income can therefore be seen to be a good indicator of the overall income in a given neighborhood.

\section{Public Transport in Madrid}

The public transport system in the Madrid metropolitan area consists of four modes, two of which are typically urban (underground rail system and urban buses), while the other two are predominantly metropolitan modes (commuter rail and interurban buses). All the transport modes are integrated into a public authority called Consorcio Regional de Transportes de Madrid (CRTM) (Madrid Regional Transport Consortium) in charge of coordinating modes and fares.

Besides the typical single and multiride (10-trip) tickets, there is a travel pass available in Madrid to promote use of public transport. The travel pass is a monthly flat fare suitable only for frequent users and represents a considerable implicit subsidy. Vassallo et al. report that, on average, travel pass users pay $35.5 \%$ less per trip than do users of typical single and multiride tickets (4). This pass is valid for 1 month on all public transport modes inside a certain ring. Travel 
pass holders can make unlimited trips inside the ring zone associated with that travel pass. There are three types of transit passes, including a regular travel pass and two special kinds of travel passes that address potentially vulnerable groups: a travel pass for young people (younger than age 23 years) and a travel pass for senior citizens (65 years and older). In 2011, young people paid 35\% less and seniors $77 \%$ less than for a regular monthly travel pass.

Since the adoption in 1987 of an integrated fare approach in Madrid, essentially based on a monthly and annual travel pass, there have been no studies aimed at quantifying the redistributive effects of subsidization at a disaggregated level, such as at the neighborhood or household level. It is worth noting that the implementation of the travel pass in Madrid can be considered a success with respect to promoting public transport usage because it has increased the number of trips by frequent users and the likelihood of attracting new ones.

However, the travel pass has had negative consequences on financial sustainability as the coverage ratio (ratio of revenues from users to operation costs) of the public transport system in Madrid has decreased steadily over the years, leading to the need for progressive increases in the amount of public subsidies.

All of the above factors highlight Madrid as a suitable study area for this type of analysis. It is crucial to evaluate whether the fare policy is effectively meeting its social objective and to determine to what extent these subsidies have promoted social inclusion with regard to income.

\section{PUBLIC TRANSPORT SUBSIDY POLICY IN MADRID: METHODOLOGY TO EVALUATE VERTICAL EQUITY}

\author{
According to Delbosc and Currie,
}

\begin{abstract}
there are two general categories of transportation equity: horizontal and vertical equity. Horizontal equity (fairness or egalitarianism) is concerned with providing equal resources to individuals or groups considered equal in ability. It avoids favoring one individual or group over another, and services are provided equally regardless of their need or ability. Vertical equity (social justice, environmental justice or social inclusion) is concerned with distributing resources among individuals of different abilities and needs. Vertical equity favors groups based on social class or specific needs in order to make up for overall societal inequalities. (16)
\end{abstract}

While both dimensions of equity can contribute to the analysis, this paper focuses on the vertical equity implications of transportation subsidy policies applied to the case study of Madrid, as there is little quantitative analysis of their distributional incidence on different income groups. This section defines the methodology that was designed to obtain the results and explains the databases used for the analysis.

\section{Data Collection}

Every 8 years the Madrid transport authority (CRTM) conducts a mobility survey in the Madrid metropolitan area that provides travel data and travel trends over time. The last survey available at the time of writing was conducted in 2004. Because there are no more recent data (the 2012 survey was canceled because of a shortage of funds), disaggregated data from the 2004 Transport Survey were used in this study. This survey enabled the authors to design a set of indicators to measure the distributional impacts at the neighborhood level (individual data are not available).
The Madrid Statistical Yearbook was used to obtain information about the characteristics of the population in each neighborhood and to study the relationship between TPU per neighborhood and the variables characterizing each one (17). In addition, income per capita data were used from the Madrid Region Statistical Institute, available at the census section level. The CRTM provided data on public transport fares, subsidies, and accessibility for each neighborhood.

Given the fact that some of these variables only have a yearly record (i.e., travel data and income levels), this study relies on crosssectional data. Consequently, all data from the Madrid Statistical Yearbook, Madrid Region Statistical Institute, and CRTM used for this analysis are from 2004. Therefore, the results are limited by the period considered and do not allow an analysis over time. Unfortunately, the lack of more recent data in Madrid makes an up-to-date analysis not possible.

Finally, the results of this research are also constrained by the aggregate nature of the data, which are collected at the neighborhood level. Accuracy would be improved by using data from a completely disaggregated survey that captures more precise individual characteristics and preferences. Unfortunately, this information is not yet available in Madrid.

\section{Some Previous Calculations: Accessibility to Public Transport in Madrid}

Accessibility is often used as a social indicator (18-21). Geurs and van Wee claim that social equity impacts can be evaluated if the accessibility measure is spatially differentiated and disaggregated (18); the authors therefore decided to look more closely at accessibility to public transport in Madrid to incorporate this variable into the present research (see the section on variables that might explain the use of the travel pass).

The approaches for measuring accessibility often include the application of geographical information systems. This research applied an approach based on the integration of existing geographical information systems and a detailed city street map to measure accessibility to transit services in different neighborhoods in Madrid. This research is the first work to explore this issue at such a disaggregated level.

Accessibility to public transport in Madrid was studied by analyzing the service area of each facility (underground rail, commuter rail, urban and interurban bus) within each neighborhood in the city. Service areas were calculated as irregular polygons depicted by using locally accepted walking distances to the transit stations, according to a Madrid City Hall plan (22). After the service areas around all the transport stations on the network street lines were computed, the accessibility to public transport for each neighborhood was obtained as the ratio of service areas (including all the stations influencing that neighborhood) to the total neighborhood area.

A statistical analysis was conducted that aimed to analyze the distribution of the accessibility values previously calculated. It was concluded that this distribution was heterogeneous and slightly skewed toward low values.

\section{Methodology for Assessing Vertical Equity Impacts}

Following the research objectives, a methodology was developed to evaluate the impact of fare subsidies on vertical equity in Madrid. Two steps were defined: the development of indicators to measure the role 
of public transportation on social equity and the study of TPU through potential explanatory variables.

\section{Indicators to Evaluate the Distributional Impacts of Public Transport Fare Policy in Madrid}

To develop these indicators, it is first necessary to select the type of equity to evaluate and the method of categorizing people. This research was based on vertical equity and categorized people by income class (lower-income areas). The study opted to prioritize the economic needs of potentially vulnerable groups over other needs to evaluate whether the subsidy policy is progressive with respect to income per capita.

Indicators were chosen with the following characteristics: easy to explain, reliable, specific, representative, and easy to validate. Two quantitative indicators were developed by monitoring the effectiveness of travel pass policy in favoring disadvantaged groups. The following list shows the indicators and how they should behave to promote vertical equity with regard to income and social class:

- TPU percentage per neighborhood. The TPU indicator is an indirect measurement of transport affordability. Vertical equity would mean that a significantly higher percentage of the population in the poorest neighborhoods would benefit from the travel pass compared with that in the wealthiest neighborhoods.

- Average user cost per trip for travel pass users across Madrid neighborhoods (ACT). The ACT indicator shows whether lowerincome people would be expected to pay less per trip than would nonvulnerable groups.

\section{Variables That Might Explain the Use of the Travel Pass}

A linear regression model was designed and fitted to explain the use of the travel pass and evaluate the distribution of transport subsidy benefits among different income groups in Madrid. A model was calibrated to estimate the relationship between a dependent variable (the number of people who buy the travel pass per neighborhood) and some explanatory variables that are assumed to influence TPU.

To conduct this statistical analysis, a database panel was built with 128 observations corresponding to the 128 neighborhoods in the Madrid metropolitan area. A model was set up to predict the use of the travel pass in each neighborhood by testing the following independent variables that characterize each of the 128 neighborhoods:

- Income per capita;

- Neighborhood area;

- Total population and potential population (defined below) for the regular pass, young people's pass, and senior travel pass;

- Percentages of population with high, medium-high, medium, medium-low, and low socioeconomic levels;

- Motorization;

- Number of people with different educational levels: illiterate, no studies, first grade or secondary education, high school, basic or higher professional training, diploma courses, bachelor's degree, and doctoral studies;

- Employed, unemployed, active population, inactive population (women and men);

- Housing price per square meter; and

- Public transport accessibility.
Since multicollinearity is a traditional problem in regression analysis and to avoid erroneous inferences from possible relationships between predictor variables, collinearity was examined between all the independent variables through the correlation matrix. If there is multicollinearity between two predictor variables, then the correlation coefficient between these variables will be nearer to 1.0. If there is a high correlation between two variables, one variable is excluded from consideration.

Multiple regression analysis was then applied to predict TPU. By constructing the full model with all the predictor variables obtained after the detection of multicollinearity, the full model $R^{2}$-value was obtained. The significance of the model's coefficients was also tested so as to discard variables with no influence on the dependent variable. After researchers started with a full model and eliminated variables according to this test, a final model was obtained in which all the coefficients were significantly different from zero. Different models were run until the optimum was found with respect to the multiple correlation coefficient $R$, the residual standard error, and a test of significance for $R$. On the basis of the results, a final examination was made of the actual signs of the linear coefficients and they were compared with their predictable signs.

The cross-validation technique was used to detect and prevent overfitting. On the basis of a 10 -fold cross-validation test, it was estimated how accurately the predictive final model would perform in practice. Finally, following econometric practice, the elasticities of independent variables in the use of the travel pass were interpreted.

\section{ANALYSIS AND FINDINGS}

This section shows the results of the indicators used to evaluate the impact of TPU on vertical equity across different neighborhoods. For the TPU indicator, the results are displayed on a map showing the indicators for the 128 neighborhoods in the city of Madrid. The neighborhoods were split into three income levels: low income (up to $€ 10,310$ per capita); medium income (between $€ 10,310$ and $€ 14,700)$; and high income (between $€ 14,700$ and $€ 31,218)(€ 1.00=$ US\$1.24 in 2004) (Figure 1). In addition, Figure 1 includes information about the population and the population densities for the 128 neighborhoods in the city.

\section{Indicators to Evaluate the Distributional Impacts of Public Transport Fare Policy in Madrid}

\section{TPU Percentage per Neighborhood}

The first indicator measures the percentage of the population using the travel pass in each neighborhood. Within each neighborhood, the potential population $\left(P_{p i}\right)$ of travel pass users was calculated by adding the potential population for the regular pass, young people's pass, and senior travel pass. As explained before, the criteria for obtaining a special travel pass depend on age, as established by the CRTM. For example, the potential population for the young people's travel pass is defined as individuals younger than age 23 years living in the neighborhood, and 65 years and older for the senior travel pass. The group of regular travel pass users is made up of individuals between the ages of 23 and 64 years.

The TPU indicator was calculated through the following ratio. The numerator of the equation was obtained from the 2004 Trans- 


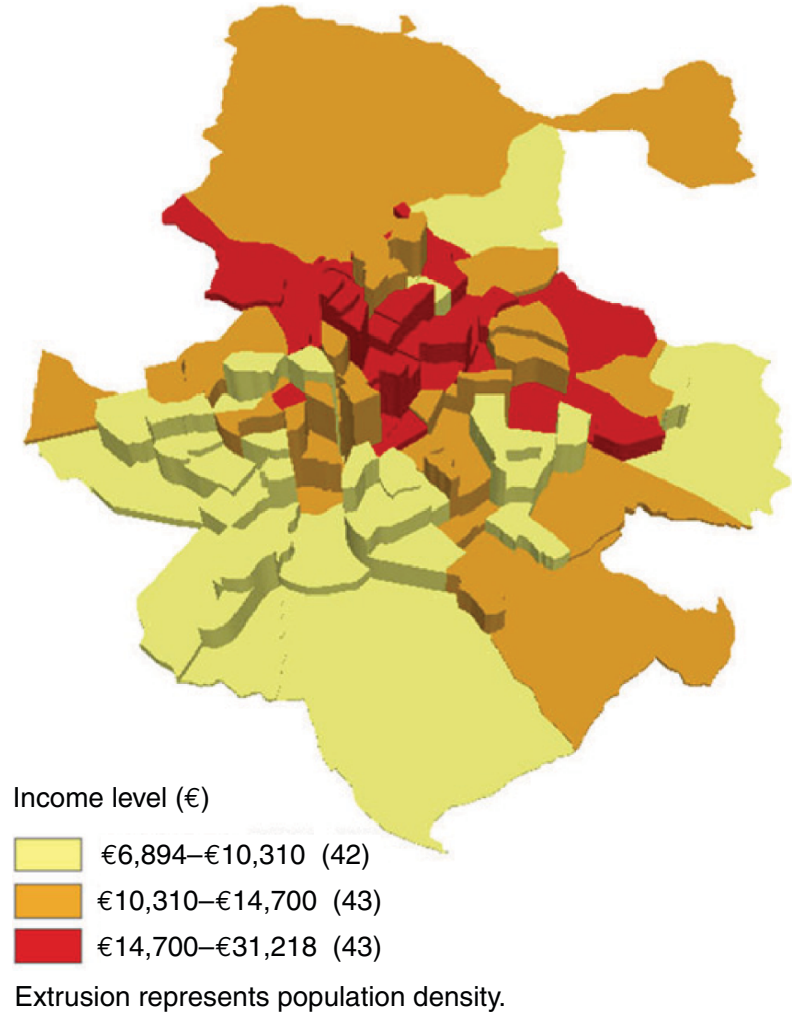

(a)

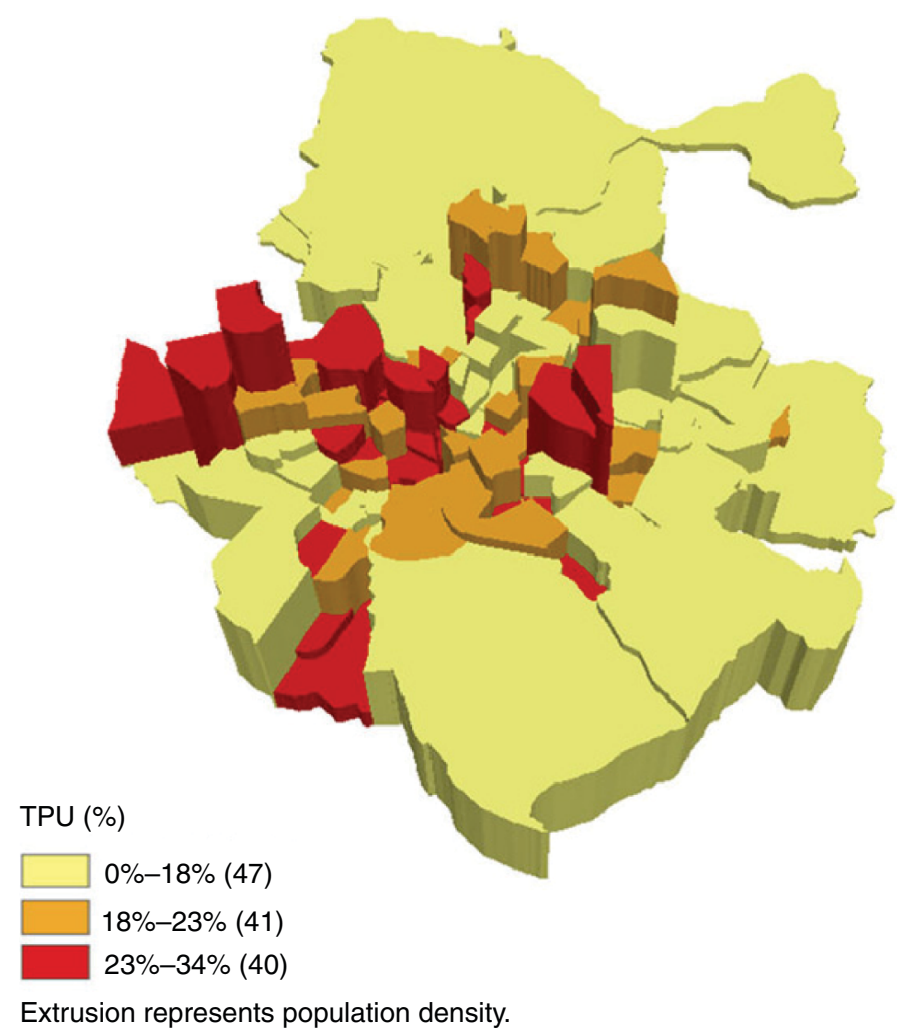

(b)

FIGURE 1 Neighborhood characteristics: $(a)$ income level and (b) TPU percentage (numbers in parentheses indicate number of Madrid neighborhoods in each category).

port Survey. The denominator is the sum of potential population for all types of travel pass per neighborhood.

$$
\operatorname{TPU}_{i}(\%)=\frac{\begin{array}{c}
\text { residents of neighborhood } i \\
\text { who actually use the travel pass }
\end{array}}{P_{p i}}
$$

General TPU values per neighborhood are shown in Figure 1. According to this indicator, there is some evidence that subsidies contribute to promoting social equity in the city, as the TPU percentage appears to be higher in the disadvantaged neighborhoods of Madrid. On the basis of the calculations of this indicator, it was found that $88 \%$ of the neighborhoods with a high TPU percentage are low-income areas. The highest TPU percentages occur in disadvantaged neighborhoods such as Campamento (TPU $=34 \%)$, Justicia $(\mathrm{TPU}=33 \%)$, and La Chopera $(\mathrm{TPU}=31 \%)$.

These results support the hypothesis that the travel pass is vertically equitable, because the highest percentages of this indicator are distributed among economically disadvantaged neighborhoods. Given that the travel pass is the most highly subsidized fare in Madrid, this public transport policy provides the greatest benefit to lower-income groups.

\section{Average User Cost per Travel Pass Trip Across Madrid Neighborhoods}

The average user cost per travel pass trip is designed to determine whether people using the travel pass in low-income neighborhoods are more subsidized than people using the travel pass in high-income neighborhoods. This methodology was applied separately for each type of travel pass user (regular pass, young people's pass and senior pass) to obtain the subsidy effect for each group. On the basis of the number of residents per neighborhood who actually use the travel pass and the fares for each type of travel pass, the amount of money they spent on transportation per month $\left(\mathrm{AMI}_{i}\right)$ was calculated. The transportation survey was used to obtain the number of monthly trips made with each type of travel pass per neighborhood and then the average user cost per trip $\left(\mathrm{ACT}_{i}\right)$ per neighborhood and type of travel pass was calculated as follows:

$$
\begin{aligned}
& \mathrm{ACT}_{i} \mathrm{RTP}(€)=\frac{\mathrm{AMI}_{i} \mathrm{RTP}}{\mathrm{TMT}_{i} \mathrm{RTP}} \\
& \mathrm{ACT}_{i} \mathrm{YTP}(€)=\frac{\mathrm{AMI}_{i} \mathrm{YTP}}{\mathrm{TMT}_{i} \mathrm{YTP}} \\
& \mathrm{ACT}_{i} \mathrm{STP}(€)=\frac{\mathrm{AMI}_{i} \mathrm{STP}}{\mathrm{TMT}_{i} \mathrm{STP}}
\end{aligned}
$$

where

$\mathrm{ACT}_{i} \mathrm{RTP}=$ average user cost per trip for residents of neighborhood $i$ using a regular travel pass,

$\mathrm{ACT}_{i} \mathrm{YTP}=$ average user cost per trip for residents of neighborhood $i$ using a young people's travel pass,

$\mathrm{ACT}_{i} \mathrm{STP}=$ average user cost per trip for residents of neighborhood $i$ using a senior travel pass, 
$\mathrm{AMI}_{i} \mathrm{RTP}=$ amount of money spent on transportation per month by residents of neighborhood $i$ using regular travel passes,

$\mathrm{AMI}_{i} \mathrm{YTP}=$ amount of money spent on transportation per month by residents of neighborhood $i$ using young people's travel passes,

$\mathrm{AMI}_{i} \mathrm{STP}=$ amount of money spent on transportation per month by residents of neighborhood $i$ using senior travel passes,

$\mathrm{TMT}_{i} \mathrm{RTP}=$ total monthly trips by regular travel pass users living in neighborhood $i$,

$\mathrm{TMT}_{i} \mathrm{YTP}=$ total monthly trips by young people's travel pass users living in neighborhood $i$, and

$\mathrm{TMT}_{i} \mathrm{STP}=$ total monthly trips by senior travel pass users living in neighborhood $i$.

To be vertically equitable, the ACT ratio should be lower in disadvantaged neighborhoods, meaning that travel pass users from poorer neighborhoods are more highly subsidized per trip than those from richer neighborhoods. However, results for the ACT ratio suggest a fairly equal treatment of all users (regardless of their income levels), which is strictly speaking not vertically equitable with respect to income. Subsidies for travel pass users do not distinguish between low- and high-income neighborhoods. High-income travel pass users pay on average $€ 0.47$ per trip with the regular travel pass, $€ 0.33$ per trip with the young people's travel pass, and $€ 0.13$ per trip with the senior travel pass. Lower-income users pay similar amounts for a trip made with the regular pass and young people's travel pass, whereas they pay $€ 0.14$ per trip with the senior travel pass.

However, there are clear differences between the average user costs per trip for regular passes, young people's passes and senior travel passes. The user cost per trip is $€ 0.47$ with a regular travel pass, €0.33 with a young people's travel pass, and $€ 0.13$ with a senior travel pass. This amount shows an equitable distribution of benefits and costs among minority groups such as young people and seniors.

The ACT indicator shows that the subsidies for travel pass users cannot be claimed to support vertical equity because they are equally distributed across neighborhoods. Nevertheless, because residents of low-income neighborhoods have a higher TPU level than those of richer neighborhoods (shown by the TPU indicator), the poorest neighborhoods still receive a higher subsidy than the richest.

A more comprehensive equity evaluation is needed to understand the causes that could explain the level of TPU in a given neighborhood. The next section shows how significant explanatory variables can be found by fitting a model.

\section{What Variables May Explain TPU?}

This section aims to identify the variables that explain the use of the travel pass across neighborhoods. A multiple regression model was used in which all the colinear variables within the set of selected variables were removed to assess whether the regression parameters were significant. If they were, the results were interpreted with respect to vertical equity.

Before calibrating the model, an evaluation was made as to whether multicollinearity was present in the data. As the linear model was correlated between the logarithms of all the variables, the correlation matrix was obtained for the logarithms of all the independent vari- ables. After the correlation matrix was inspected, it was decided to remove the following variables because they were highly correlated with the rest of the variables (shown in parentheses) and did not provide any further information to the linear model:

- Potential populations for the regular pass, young people's pass, and senior travel pass (very highly correlated with overall population);

- Percentages of population with high, medium-high, medium, medium-low, and low socioeconomic status (highly correlated with population);

- Motorization (very highly correlated with population and number of people with different educational levels);

- Number of people with different educational levels (highly correlated with available income);

- Employed population, unemployed population, active population, and inactive population-women and men (highly correlated with population); and

- Housing price per square meter (correlated with available income).

In summary, from all the possible groups of noncollinear variables, the set of income, area, population, and accessibility was selected. As has been widely discussed in social research studies $(6,18,23,24)$, these variables were considered in the analysis to provide an adequate explanation of public transport use from an equity standpoint. Population and area were included as control variables. Because of its nature, public transportation use is probably highly influenced by income; a low income may contribute to disadvantages with regard to transportation. Finally, the heterogeneity found in public transportation coverage in different areas of the city suggests that accessibility may also play a key role in explaining public transportation demand.

After the colinear variables were removed, a multiple regression model was calibrated with the variable "number of travel passes acquired by people from a certain neighborhood" as the dependent variable. It was necessary to transform the set of variables before fitting the model since their relationship was unlikely to be linear. The Box-Cox transformation for linear models was used (25), which suggested using a logarithmic transformation. The correlation matrix explained in the previous paragraph was also performed with logarithmic transformations.

Once the transformed variables were obtained, a linear model was calibrated by using multiple regression. In this model, not all the variables had coefficients significantly different from zero. The linear model was repeated, removing the nonsignificant neighborhood area variable, and a model was finally calibrated on the basis of three endogenous variables: income per capita in each neighborhood, population, and accessibility. The correlation coefficient was strong (multiple $R^{2}=.733$; adjusted $R^{2}=.7265$; and $p$-value near zero). The results show that the use of the travel pass in Madrid is very well explained by only these three independent variables: income per capita in the neighborhood, total population, and the level of public transport accessibility (Table 1 ).

The final model shows a good overall level of fit and all the regression parameters were significant predictors (two of the variables at the $99 \%$ confidence level and the other at $90 \%$ ), so there is strong statistical evidence supporting these relationships. Table 1 also shows the comparison between the signs expected and obtained for the regressions. All the signs were in line with the expected results. For instance, as was hypothesized, a negative sign was found for the variable "income 
TABLE 1 Model Results

\begin{tabular}{lccccc}
\hline $\begin{array}{l}\text { Independent Variable } \\
\text { per Neighborhood }\end{array}$ & $\begin{array}{l}\text { Coefficient of } \\
\text { Elasticity }\end{array}$ & SE & $\begin{array}{l}\text { Sign } \\
\text { Expected }\end{array}$ & $\begin{array}{l}\text { Sign } \\
\text { Obtained }\end{array}$ & $p$-Value \\
\hline Income level & -0.68 & 0.16 & - & - & $p \sim 0^{* * *}$ \\
Population & 1.04 & 0.10 & + & + & $p \sim 0^{* * *}$ \\
Accessibility & 0.30 & 0.12 & + & + & $p \sim 0^{*}$ \\
\hline
\end{tabular}

NoTE: $\mathrm{SE}=$ standard error.

$* * * 99 \%$ confidence level; $* 90 \%$ confidence level.

level," meaning that higher levels of neighborhood income are related to lower use of the travel pass. The same applies to "accessibility," with a positive coefficient in this case.

Finally, the predictive equation can be expressed as follows:

$\log \left(\mathrm{TPU}_{i}\right)=2.94-0.68 \log$ income $_{i}+1.04 \log$ population $_{i}$

$+0.30 \log$ accessibility $_{i}$

where

$$
\begin{aligned}
\mathrm{TPU}_{i} & =\text { number of travel pass users in neighborhood } i, \\
\text { income }_{i} & =\text { income per capita in neighborhood } i, \\
\text { population }_{i} & =\text { total population in neighborhood } i, \text { and } \\
\text { accessibility }_{i} & =\text { public transport accessibility in neighborhood } i .
\end{aligned}
$$

Since the predictive equation was estimated by using a logarithmic transformation for all the variables (including the dependent variable), the estimated coefficient can be interpreted as the elasticity. In this functional form, from the double log equation (Equation 5), $-0.68,1.04$, and 0.30 are the elasticity coefficients for the income level, population, and accessibility variables, respectively. This result means that, other things being equal, a $10 \%$ increase in income level will generate a $6.8 \%$ decrease in TPU level per neighborhood, whereas a $10 \%$ increase in accessibility will generate a $3.0 \%$ increase.

Finally, the internal validity of the model was tested by means of a 10-fold cross-validation method. The neighborhoods were divided into two sets: a training set and a test set. The training set contains $90 \%$ of the neighborhoods and was used to fit the linear model. The model was then applied to the test set (i.e., 10\% of the remaining neighborhoods). Then the mean squared relative error (MSRE) of the prediction of the test set was evaluated. This procedure was repeated 5,000 times, with randomly selected training and test sets. The median error for this simulation was $4.5 \%$. The average error was $31.3 \%$ with a standard deviation of $69.2 \%$. The error distribution is highly skewed, which explains the difference between the median and average values. Most of the simulations had a very low MSRE value, but in some specific cases the MSRE value was very high. Figure 2 shows a box plot of the errors of this simulation with 5,000 random sets. Most of the models had an MSRE value of lower than $5 \%$. This test is further evidence that this model can accurately predict TPU levels.

To summarize, it was found that the use of the travel pass in Madrid is fairly well explained by variables such as accessibility to public transport and income level. The level of accessibility has a positive effect on TPU levels, whereas income level has a negative influence. In other words, the use of the travel pass and its subsidy benefits increase when the average income per capita in the neighborhood decreases. Moreover, the elasticity values provide evidence that public transport use in Madrid is more sensitive to income variations than to accessibility. These results confirm that public transport subsidies in Madrid tend to be progressive with respect to income. Consequently, the current subsidy policy in regard to TPU in Madrid can be claimed to benefit lower-income areas.

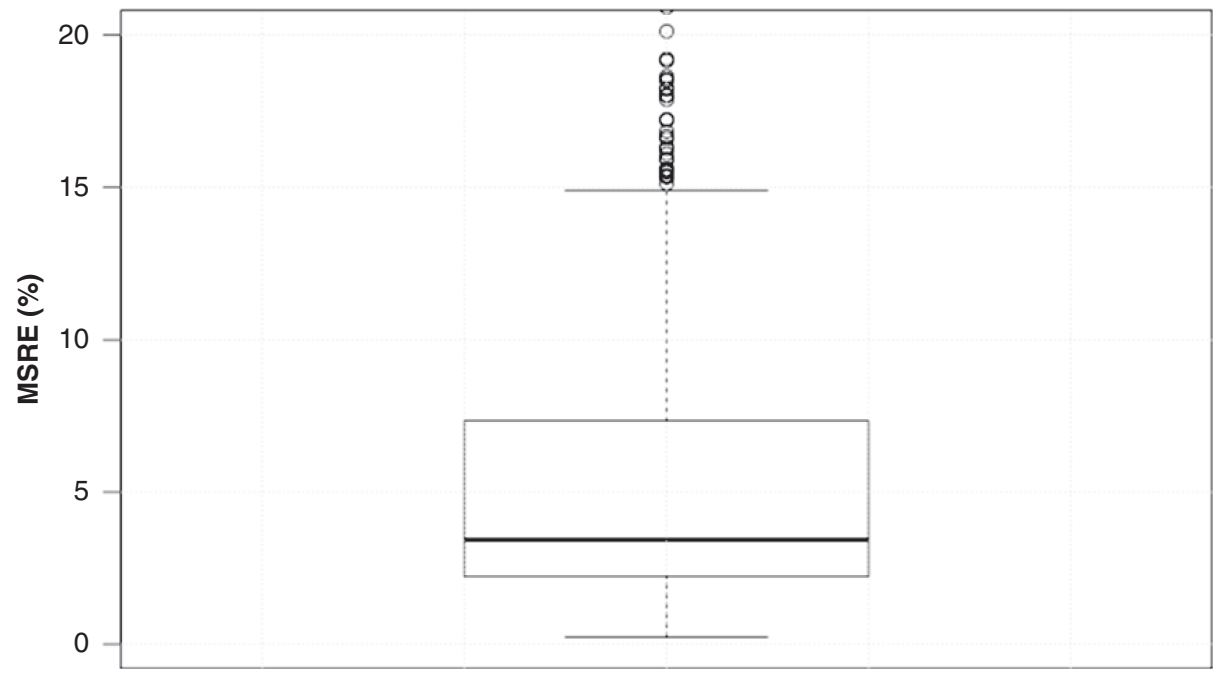

FIGURE 2 Cross-validation results. 


\section{CONCLUSIONS}

This research highlights some interesting conclusions about the effect of public transport fare and subsidy policy in Madrid on vertical equity. The TPU indicator confirms that people in poorer neighborhoods use travel passes much more often than do people in richer areas and thus obtain greater benefit from public subsidies. The results are categorical enough to confirm that public transport subsidies give special consideration to poor neighborhoods compared with wealthy ones. This indicator reveals that the travel pass subsidy policy satisfactorily targets the least wealthy people. However, the ACT indicator shows that travel pass users' cost per trip varies slightly across neighborhoods and that travel pass users in the lowest-income neighborhoods pay the same amount per trip as in high-income neighborhoods.

The multiple regression model clearly explains TPU with respect to three variables: population, accessibility, and income level. The results show that income level plays the most significant role in influencing public transport use. The lower the income level of the neighborhood, the greater is the use of the travel pass. When two neighborhoods in Madrid with the same standards of accessibility were compared, it was found that people in the poorer neighborhoods used the travel pass more than did people in the wealthier neighborhoods. These results support the claim that the subsidy policy in Madrid is progressive, since it establishes a fairness of treatment between individuals with different income levels. This finding means that vertical equity principles are fulfilled insofar as the transport policy favors economically disadvantaged groups.

Since there is very little analysis of the distributional incidence of public transport subsidies in the city of Madrid, local transport planners should take advantage of these findings to evaluate the effectiveness of the policy in reaching lower-income citizens. This analysis can also alert policy makers, practicing planners, scholars, and citizens in other contexts on the need to determine the progressiveness of targeting subsidies to the poor.

\section{REFERENCES}

1. Geurs, K., W. Boon, and B. van Wee. Social Impacts of Transport: Literature Review and the State of the Practice of Transport Appraisal in the Netherlands and the United Kingdom. Transport Reviews, Vol. 29, No. 1, 2009, pp. 69-90.

2. Beyazit, E. Evaluating Social Justice in Transport: Lessons to Be Learned from the Capability Approach. Transport Reviews, Vol. 31, No. 1, 2011, pp. 117-134.

3. Nuworsoo, C., A. Golub, and E. Deakin. Analyzing Equity Impacts of Transit Fare Changes: Case Study of Alameda-Contra Costa Transit, California. Evaluation and Program Planning, Vol. 32, No. 4, 2009, pp. 360-368.

4. Vassallo, J.M., P. Pérez De Villar, R. Muñoz-Raskin, and T. Serebrisky. Public Transport Funding Policy in Madrid: Is There Room for Improvement? Transport Reviews, Vol. 29, No. 2, 2009, pp. 261-278.
5. Serebrisky, T., A. Gómez-Lobo, N. Estupiñán, and R. Muñoz-Raskin. Affordability and Subsidies in Public Urban Transport: What Do We Mean, What Can Be Done? Transport Reviews, Vol. 29, No. 6, 2009, pp. 715-739.

6. Litman, T. Evaluating Transportation Equity. World Transport Policy and Practice, Vol. 8, No. 2, 2012, pp. 50-65.

7. Burton, E. The Compact City: Just or Just Compact? A Preliminary Analysis. Urban Studies, Vol. 37, No. 11, 2000, pp. 1969-2006.

8. Bureau, B., and M. Glachant. Distributional Effects of Public Transport Policies in the Paris Region. Transport Policy, Vol. 18, No. 5, 2011, pp. 745-754.

9. Pucher, J. Equity in Transit Finance: Distribution of Transit Subsidy Benefits and Costs Among Income Classes. Journal of the American Planning Association, Vol. 47, No. 4, 1981, pp. 387-407.

10. Guria, J.C., and A.E. Gollin. Net Tax Incidence for Urban Public Transit Subsidies in New Zealand. Transportation, Vol. 13, No. 4, 1986 , pp. 319-328.

11. Gómez-Lobo, A. A New Look at the Incidence of Public Transport Subsidies. Journal of Transport Economics and Policy, Vol. 43, No. 3, 2009, pp. 404-425.

12. Asensio, J., A. Matas, and J. L. Raymond. Redistributive Effects of Subsidies to Urban Public Transport in Spain. Transport Reviews, Vol. 23, No. 4, 2003, pp. 433-452.

13. Garcia-Ferrer, A., M. Bujosa, A. de Juan, and P. Poncela. Demand Forecast and Elasticities Estimation of Public Transport. Journal of Transport Economics and Policy, Vol. 40, No. 1, 2006, pp. 45-67.

14. Matas, A. Demand and Revenue Implications of an Integrated Public Transport Policy: The Case of Madrid. Transport Reviews, Vol. 24 No. 2, 2004, pp. 195-217.

15. Monzón, A., and M. J. Guerrero. Valuation of Social and Health Effects of Transport-Related Air Pollution in Madrid (Spain). The Science of the Total Environment, Vol. 334, 2004, pp. 427-434.

16. Delbosc, A., and G. Currie. Using Lorenz Curves to Assess Public Transport Equity. Journal of Transport Geography, Vol. 19, No. 6, 2011, pp. 1252-1259.

17. Madrid Statistical Yearbook. Madrid City Hall, Department of Statistics, Madrid, Spain, 2004.

18. Geurs, K. T., and B. van Wee. Accessibility Evaluation of Land-Use and Transport Strategies: Review and Research Directions. Journal of Transport Geography, Vol. 12, No. 2, 2004, pp. 127-140.

19. Shen, Q. Spatial Technologies, Accessibility, and the Social Construction of Urban Space. Computers, Environment and Urban Systems, Vol. 22, No. 5, 1998, pp. 447-464.

20. Wachs, M., and T. G. Kumagai. Physical Accessibility as a Social Indicator. Socio-Economic Planning Sciences, Vol. 7, No. 5, 1973, pp. 437-456.

21. Bocarejo, J.P., and D.R. Oviedo. Transport Accessibility and Social Inequities: A Tool for Identification of Mobility Needs and Evaluation of Transport Investments. Journal of Transport Geography, Vol. 24, 2012, pp. 142-154.

22. Madrid City Hall. Sustainable Urban Mobility Plan of the Madrid Center District. Department of Transportation, Madrid, Spain, 2013.

23. Currie, G. Quantifying Spatial Gaps in Public Transport Supply Based on Social Needs. Journal of Transport Geography, Vol. 18, No. 1, 2010, pp. 31-41.

24. Delbosc, A., and G. Currie. Transport Problems That Matter-Social and Psychological Links to Transport Disadvantage. Journal of Transport Geography, Vol. 19, No. 1, 2011, pp. 170-178.

25. Box, G. E., and D. R. Cox. An Analysis of Transformations. Journal of the Royal Statistical Society, Vol. 26, No. 2, 1964, pp. 211-252.

The Standing Committee on Public Transportation Marketing and Fare Policy peer-reviewed this paper. 